\title{
Trajectory-based worker task productivity monitoring
}

\author{
E. Konstantinou ${ }^{\mathrm{a}}$ and I. Brilakis ${ }^{\mathrm{b}}$ \\ ${ }^{a}$ Department of Engineering., University of Cambridge, UK \\ ${ }^{b}$ Department of Engineering., University of Cambridge, UK \\ E-mail: ek415@cam.ac.uk,ib340@cam.ac.uk
}

\begin{abstract}
Over the past decades labour productivity in construction has been declining. The prevalent approach to estimating labour productivity is through an analysis of the trajectories of the construction entities. This analysis typically exploits four types of trajectory data: a) walking path trajectories, b) dense trajectories (posture), c) physiological rates such as heart rate (beats/minute) and respiratory rate (breaths/minute), and d) sound signals. The output of this analysis is the number of work cycles performed by construction workers. The total duration of these cycles is equal to the labour input of a task. However, all such methods do not meet the requirements for proactive monitoring of labour productivity in an accurate, non-obtrusive, time and cost efficient way for multiple workers. This paper proposes a method to address this shortcoming. It features a promising accuracy in terms of calculating the labour input.
\end{abstract}

Keywords - Productivity, monitoring, construction.

\section{Introduction}

In general, productivity is defined as the ratio of output to input [1]. Productivity rates are used by project managers during planning and scheduling in order to reduce the labour cost and improve the performance of workers. The construction sector has gradually created a significant labour productivity gap compared to other industries over the past five decades. It is estimated that only $50 \%$ of the total construction time is productive $[2,3]$. This is due to factors that affect on-site construction tasks negatively. Almost all of them are related to the way that productivity is monitored. Construction project managers currently evaluate worker performance based on questionnaires, manual observations, and work sampling practices [4-7]. Construction requires proactive monitoring of labour productivity in order to detect issues sufficiently early. However, this is not feasible as current practices are labour intensive and time consuming due to the large number of employees and the long lasting tasks. This paper presents a method to address this issue.
The remainder of this paper is structured as follows. Sections 2 analyses the current state of research in monitoring of labour productivity in construction. Section 3 presents an overview of the overall proposed method presented in this paper. Section 4 summarizes the outcomes of this paper.

\section{Background}

This paper reviews the latest studies that focus on monitoring of labour productivity. Current studies are divided in two main categories based on the methods they employ to infer productivity. The first contains the region-based studies that link the location of workers to regions of management interest (work zones) such as steel fixing zone, concrete pouring zone. The second consists of the activity-based studies that detect and link activities such as bending, hammering, and drilling to specific tasks.

Region-based studies monitor labour productivity through the time construction entities spend at zones of management interest (e.g. excavation zone, concrete pouring zone). In order to achieve this, the location of monitored entities is tracked across the jobsite. The studies of this category are sub-divided into tagged and tag-less. The tagged (RF tagged) studies employ tags which are physically attached on workers and earthmoving equipment. The most frequently used tags are the Global Positioning System (GPS), the Radio Frequency Identification system (RFID), and the UltraWide band system (UWB). The above systems provide the input data of tagged studies. On this basis, the speed and the location of a haul truck were both combined for monitoring its productivity while performing an earthmoving operation [8]. If the haul truck's location was within the range of fixed known distances from specific work zones (e.g. load and dump zones), then the time during which its speed was equal to zero was converted into labour input. On the other hand, the labour productivity of workers, was monitored by linking their presence at predefined work zones [9-13]. For instance, if a concrete worker is located at zones " $\mathrm{A}$ " and " $\mathrm{B}$ " which are scheduled for concrete pouring, then the total time the worker spent in these zones is 
considered productive and equal to his/her labour input. The studies of this category also sub-divide the areas between the actual work zones into waiting and travelling zones, for a more detailed insight of worker productivity. The most important disadvantage of the tagged studies is that they can neither identify the unproductive time (idle time) nor the low productivity pace. For example, even if a worker is located in the correct work zone, but without performing any task due to shortage of materials or congestion he/she will be still considered productive. This is due to the fact that labour productivity is monitored only based on the presence of workers at work zones. The tagged studies do not provide any extra information about what really happens within these work zones. In addition, the purchase and maintenance of multiple tags impose a regular cost in the long term [14]. Last but not least, the physical attachment of tags creates a feeling of discomfort to workers [15]. The tag-less studies rely on computer vision-based 2D tracking methods in order to calculate the location of workers. This location is 2D instead of 3D. Therefore, entities are tracked only within the range of a camera's view. This type of tracking is non-obtrusive as it processes video data collected through surveillance cameras used for security purposes. The studies in this category convert the location data into labour productivity through two approaches. The first, links the presence of tracked entities (workers, earthmoving equipment) to specific work zones similar to tagged studies [16]. For this reason, the ambiguity about what really happens within these zones arises again. The second, fits the monitored entities to operation process models [17-19]. Such models [20,21]: a) break down the construction tasks into sub-tasks (semantic context), b) describe how the sub-tasks relate to specific work zones across the jobsite (spatial context), and c) define the sequential order (i.e. workflow) between the sub-tasks (temporal context). However, such approach relies on human intervention in order to adjust the appropriate process model to each entity. It takes 5-10minutes for an operator to achieve this. Such adjustments should be repeated for every entity on a daily basis. The large numbers of workers and earthmoving equipment entails that such type of studies will be labour intensive if applied in practice.

The activity-based studies firstly detect and secondly link activities to specific construction tasks in order to monitor labour productivity. These activities are the physical description of tasks. For example, a brick layer bends to pick up bricks and stretches his arms to place them. Bending and stretching are both activities that describe the brick laying task. This type of studies exploit posture, physiological (e.g. heart, breathing rate) and audio data. The posture-based studies have been used for monitoring both the labour productivity of earthmoving equipment [22,23] and construction workers [22-26]. Posture data are detected via feature descriptors such as the Histogram of Oriented Gradients [27] and skeletisation algorithms [28]. Machine learning-based algorithms such as Support Vector Machine Classifiers (SVMs) [29] and Artificial Neural Networks (ANNs) [30] are then trained to link (label) the detected activities to construction tasks. The highest achieved accuracy so far is equal to 59\% [24]. In particular, this study was tested on workers while performing 11 types of tasks i.e. brick laying, transporting, plate cutting, drilling, re-bars fixing, nailing, plastering, shovelling, bolting, welding, and sawing. The authors admitted that this low accuracy was due to the fact that most of these tasks were not distinguishably described by posture data. On the other hand, posture-based studies perform very well (accuracy $>80 \%$ ) for the case of earthmoving equipment, as such entities have a small but well defined range of postures. For example, an excavation task performed by a dump truck is described only by two postures. The first depicts the unloading of materials and the second the transportation of materials. In addition, earthmoving equipment is used for only one type of tasks whereas workers perform a much larger variety. Physiological data such as heart rate (beats/minute), breathe rate (breaths/minute), body's force and angular rate [31-33] are acquired through physiological status monitoring (PSMs) and inertial measurement unit (IMU) wearable sensors. The physiological data are used for training machine learning-based classification methods similarly to the studies that exploit posture data. However, it has been proven that heart and breathe rates cannot establish any relationship with individual's labour productivity [31]. On the other hand, body's force and angular rate, extracted with accelerometers and gyroscopes of IMUs sensors, achieved a promising performance $(\approx 80 \%$ accuracy) in terms of detecting and labelling activities such as hammering, sawing, turning a wrench, loading/unloading/pushing a wheelbarrow [32]. Physiological-based studies have been also successfully used for identifying abnormalities in the performance of workers (awkward postures) for health and safety purposes [33]. Their main limitation is that they rely on data collected with wearable sensors that give rise to privacy issues. Lastly, audio data which are recorded by microphones placed at construction jobsites have also been exploited for monitoring the productivity of construction entities [34,35]. These audio-based studies are applicable only to tasks that produce discrete sounds such as nailing, hammering, excavating, and drilling. Although they have managed to successfully remove background noise, they are still not designed to monitor the labour productivity of multiple entities that perform similar tasks simultaneously. 


\section{Methodology}

Figure 1 illustrates a method for monitoring labour productivity of multiple workers at the same time. The skewed parallelogram shapes refer to methods and the circular to inputs/outputs. The method consists of two main sub-methods illustrated with black coloured skewed parallelogram shapes. The output of the first is the input for the second. The inputs of the method are video data streamed from multiple cameras, whilst the output of the method is the total productive and unproductive time spent by each worker. This paper hypothesizes that task productivity of construction workers can be monitored through their trajectory data.

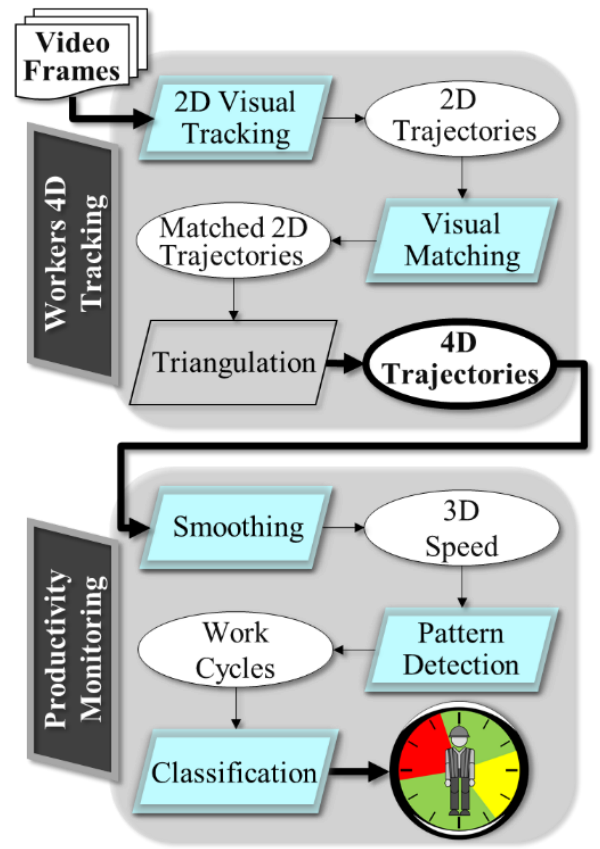

Figure 1. Method for automated construction worker task productivity monitoring.

The labour productivity is calculated by dividing a worker's total output over the total input [5]. The determination of output is quite straightforward through visual inspections (e.g. number of pipes installed, number of $\mathrm{m}^{3}$ being excavated). Hence, this paper focuses only on the input. The main assumption of this paper is that all construction-related tasks fit to the same pattern. This pattern dictates that if a worker's "move" is followed sequentially by one "stop" and a second "move", then these three semantic events define a work cycle. This assumption is based on the fact that workers "stop" in order to perform a construction-related task and they "move" to start another. In construction, a work cycle is defined as the total time a worker spends on a task [4]. Hence, the duration of a work cycle is equal to the duration of the semantic "stop" event. Sequentially, the duration of all work cycles is equal to the labour input of a worker. Therefore, the labour input can be extracted by detecting these work cycles.

The first method of the proposed method is a computer vision-based method for 4D tracking of construction workers. This type of tracking is unobtrusive as it is tag-less. The input data are videos collected through the cameras of jobsites' surveillance systems. It returns one 4D trajectory for every worker as output. These 4D trajectories depict the 3D (X, Y, and Z) location of workers across the entire range of a jobsite over time. This 4D localization overcomes the limitation of previous tag-less studies that monitored workers only within a camera's view. An intra and an inter camera tracking are performed sequentially in order to achieve this 4D tracking [36]. The former matches workers under the same unique ID across subsequent frames of a camera, whilst the latter matches workers across multiple cameras. Then, a triangulation method [37], is applied in order to convert the 2D trajectories into 4D.

The second method of the proposed method is productivity monitoring. It uses the output of the 4D tracking method as input. Initially, a smoothing method removes the noise from the $4 \mathrm{D}$ trajectories. Then, the 4D trajectory of each worker is partitioned into smaller 4D sub-trajectories. The 3D speed values of these partitions are exploited to cluster them into work cycles based on the main assumption of this paper. The accurate detection of these work cycles addresses the second aim of this paper as their total duration is equal to the labour input of construction workers. The 3D speed values depict the motion of workers along the floor (XZ) and the vertical plane (Y). The detected work cycles are classified as: a) unproductive, b) normal productive, and c) abnormal productive. Initially, they are classified as either productive or unproductive through region-based classification that splits the jobsite into two types of areas, "active" and "inactive". The former contains the areas of the jobsite where tasks such as excavation, brick laying are performed. The latter consists of areas where no construction-related tasks take place. These are the: a) rest areas, b) materials' storage areas, and c) office areas. The work cycles that take place at "active" areas are classified as productive while those that take place at "inactive" areas are classified as unproductive. Then, the productive work cycles are further classified in order to detect potential abnormalities in the pace of the labour input. The durations of the productive work cycles are compared for this purpose. Those with the highest duration are classified as potentially abnormal and the rest as normal. This second classification is used as an indicator. It shows project managers whether something appears to 
be "wrong" with workers' productivity pace. Managers can then look into the video data at the time of the day the abnormalities occurred and check whether something was actually incorrect with these work cycles. This way problems are identified and treated fast. The productivity monitoring method does not need any prior knowledge about the type or the number of tasks workers perform. Therefore, labour productivity of multiple workers can be monitored at the same time. This entails proactivity.

\section{Results}

This section evaluates the performance of the method presented in this paper in terms of translating the trajectory data into labour input. This is achieved with a C\# implementation in Microsoft Visual Studio.Net framework running in a Windows 8.1 operating system. The integrated development environment is Visual Studio 2013, using Windows Forms (WinForms). A desktop PC with the following specs is used: Intel core i7 CPU, $4.0 \mathrm{GHz}$, and $32 \mathrm{~GB}$ RAM. The cameras used in the experiments are two GoPro cameras, black edition 4 with a 1920x1080 frame size, and selected $90^{\circ}$ narrow field of view to reduce the distortion of camera fish eye effect. Both cameras are mounted in such a way that monitored workers are captured within their overlapping field of views.

Precision, recall, and accuracy metrics are used for the evaluation of this chapter's proposed method. Precision is the fraction of the total number of correctly detected work cycles (TP, True Positive) over the total number of incorrectly and correctly detected work cycles (TP + FP, True Positive + False Positive). Recall depicts the detection completion level and is equal to the total number of correctly detected work cycles (TP) divided by the total number of correctly detected and incorrectly not detected work cycles $(\mathrm{TP}+\mathrm{FN}$, True Positive + False Negative). Lastly, accuracy is defined by the number of correctly detected work cycles (TP) and the number of work cycles which were correctly not detected (TN, True Negative), over the total sum of work cycles.

The proposed method is tested on an electrical task (see Figure 2). This data set consists of two recordings (part A, B) with a duration of approximately 17 minutes each. Both recordings were collected the same day. In total, steel worker performed 29 work cycles that depict the following sub-tasks: a) fixing steel re-bars, b) picking re-bars or equipment, and c) reading drawings. Only one is unproductive, whilst none of the productive work cycles corresponds to idle time.

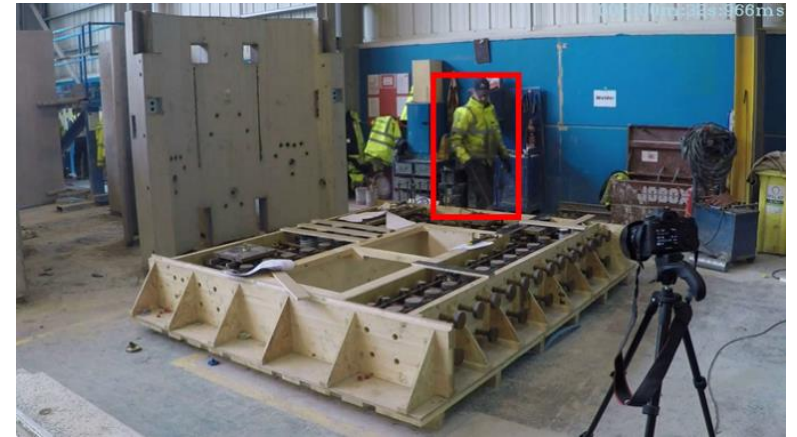

Figure 2. Tested data set.

This section colours red the unproductive, yellow the abnormal productive and green the normal productive work cycles. Figure 3 illustrates the 4D trajectories of part B.

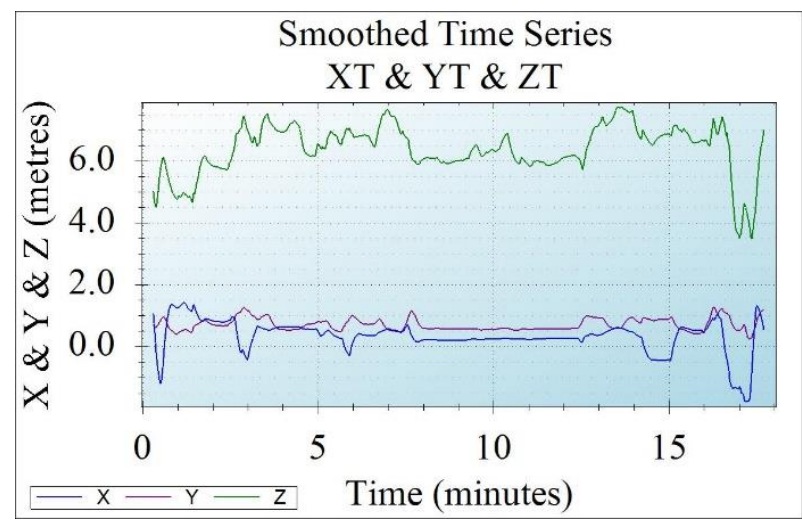

Figure 3. X, Y, Z trajectories over time (part B).

The proposed method detects $9 \mathrm{TP}, 1 \mathrm{TN}, 0 \mathrm{FP}$ and 0 FN work cycles in part A, and 10 TP, 2 TN, 7 FN and 1 FP work cycles in part B. Table 1 shows the ground truth of the manually collected work cycles vs the automated detected ones. The $3 \mathrm{TN}$ results (\#1, \#11, and $\# 12$ ) result from the way trajectories are smoothed. The smoothing step $\mathrm{k}$ is equal to $19 \mathrm{sec}$ if divided by camera frame rate i.e. $\frac{k}{f p s}=\frac{570}{30}$. Hence, all smoothed time series are $19 \mathrm{sec}$ shorter in length at the beginning compared to the unsmoothed. Hence, work cycles that fall within the initial $19 \mathrm{sec}$ cannot be detected. All TN results occur at the beginning of each recording. The missed \#17 and the 1FP work cycles of part B are due to instabilities of the implemented computer vision-based 2D tracking method. All the rest FN work cycles are of short duration $(<4 \mathrm{sec})$. This shows that that the proposed method does not perform well in terms of detecting work cycles of such short duration. 
Table 1: Manually collected ground truth of semantic "stops".

\begin{tabular}{lc}
\hline & Part A (GT) \\
$\#$ & Start - End \\
\hline 1. & $00: 00: 033-0: 05: 105(\mathrm{TN})$ \\
2. & $00: 12: 645-03: 37: 884(\mathrm{TP})$ \\
3. & $03: 39: 153-07: 48: 035(\mathrm{TP})$ \\
4. & $07: 50: 036-11: 41: 067(\mathrm{TP})$ \\
5. & $11: 42: 268-11: 44: 070(\mathrm{TP})$ \\
6. & $11: 52: 545-15: 31: 798(\mathrm{TP})$ \\
7. & $15: 35: 101-15: 40: 273(\mathrm{TP})$ \\
8. & $15: 45: 645-16: 49: 609(\mathrm{TP})$ \\
9. & $15: 57: 317-17: 02: 989(\mathrm{TP})$ \\
10. & $17: 11: 131-17: 42: 962(\mathrm{TP})$ \\
\hline & Part B (GT) \\
$\#$ & Start - End \\
\hline 11. & $00: 00: 033-00: 13: 680(\mathrm{TN})$ \\
12. & $00: 19: 919-00: 21: 287(\mathrm{TN})$ \\
13. & $00: 25: 258-00: 30: 697(\mathrm{TP})$ \\
14. & $00: 33: 867-00: 34: 768(\mathrm{FN})$ \\
15. & $00: 37: 771-01: 24: 150(\mathrm{TP})$ \\
16. & $01: 28: 855-02: 36: 322(\mathrm{TP})$ \\
17. & $02: 40: 293-02: 47: 667(\mathrm{FN})$ \\
18. & $02: 49: 202-02: 51: 071(\mathrm{FN})$ \\
19. & $02: 52: 205-05: 53: 687(\mathrm{FN})$ \\
20. & $05: 55: 021-14: 14: 087(\mathrm{TP})$ \\
21. & $14: 15: 388-15: 02: 268(\mathrm{FN})$ \\
22. & $15: 03: 670-16: 15: 175(\mathrm{TP})$ \\
23. & $16: 18: 645-16: 28: 988(\mathrm{TP})$ \\
24. & $16: 32: 359-16: 42: 102(\mathrm{TP})$ \\
25. & $16: 47: 173-17: 02: 422(\mathrm{TP})$ \\
26. & $17: 05: 658-17: 08: 628(\mathrm{FN})$ \\
27. & $17: 09: 896-17: 18: 171(\mathrm{TP})$ \\
28. & $17: 23: 943-17: 29: 416(\mathrm{FN})$ \\
29. & $17: 30: 517-17: 41: 227(\mathrm{TP})$ \\
\hline &
\end{tabular}

In Part A, the work cycle \#3 shows that steel worker was unproductive i.e. definitely not performing the steel fixing task, for 4.15 minutes. Work cycles \#5, and \#7 to \#10 are all classified as normal productive whilst the work cycles with the largest duration \#2, \#4, and \#6, are all classified as abnormal productive (see (a) Figure 4). In part B, nine out of ten work cycles of this recording, are returned as normal (\#13, \#15, \#16, \#22 to \#25, \#27 and \#29) and only one is classified as abnormal (\#20) (see (b) in Figure 4). The interesting observation about the recording of part $\mathrm{A}$, is that the abnormal cycle has a duration of 11.56 minutes which is by far the largest compared to the rest cycles of both parts A and B. This raises an ambiguity about the performance of steel worker during this time. It can be easily observed, if we check the video footages at the exact time the abnormal cycle \#20 occurred, that steel worker could not fit a reinforcing steel bar in the formwork due to complexity of drawings. This is a common issue that affects labour productivity. If we sum all the TP normal and abnormal work cycles, then the labour input of the steel worker is equal to 28.29 minutes for both parts $(\mathrm{A}, \mathrm{B})$. The manually calculated labour input is equal to 30.62minutes. Therefore, the proposed method measured the total labour input of the steel worker with an accuracy of $92 \%$.

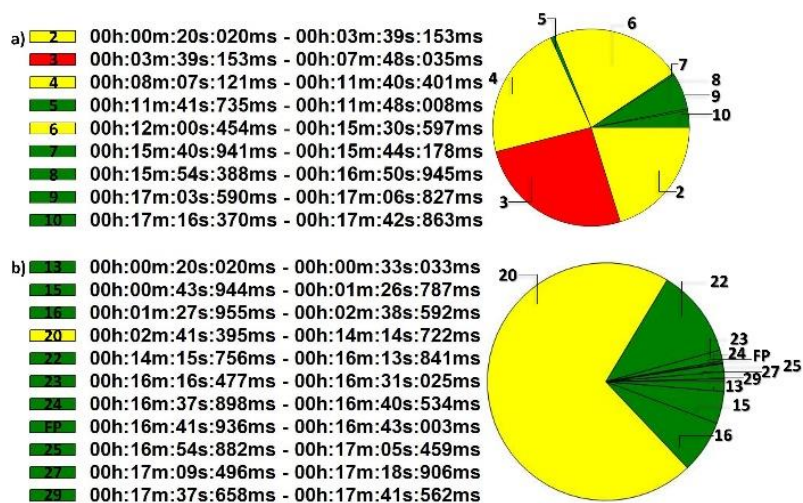

Figure 4: Detected work cycles of steel worker (red: unproductive, yellow: abnormal productive and green: normal productive).

\section{Conclusions}

The current state of research has not yet proposed a method that performs a non-obtrusive, accurate, cost efficient and generalized monitoring of labour productivity for construction workers. This paper presents a method that addresses these issues in order to detect repetitive patterns in the trajectories of construction workers that depict work cycles. The total duration of these work cycles is equal to the labour input of workers. The novelty of the proposed method lies in clustering. Firstly, the 4D trajectories of workers are smoothed in order to remove noise. Then, they are segmented into 4D sub-trajectories and classified as either "move" or "stop" semantic events. The former event depicts the motion of workers along the floor plane, whilst the latter depicts the motion of workers along the vertical plane. The classified 4D subtrajectories are finally grouped into clusters based on the main assumption of this paper that: every work cycle is described by two semantic "move" events and one semantic "stop" event.

The main limitations of the method presented in this paper are the following. Firstly, work cycles that depict workers who while at "stop" do not perform any task (idle time) are mistakenly detected as productive. However, as previous studies [38-43] stated "idle" time 
is not of the main causes behind low labour productivity. Secondly, the productivity of workers who perform tasks mainly characterized by motion such as transferring materials, supervising work progress etc. cannot be monitored. This is because the "move" events depict the actual labour input instead of the "stop" events in such cases. This second limitation indicates that the automated monitoring of workers presented in this paper cannot be applied to the entire range of construction related tasks. Only if the proposed method was updated with the type of tasks of workers it would be possible to turn also the detected "move" events into labour input.

\section{Acknowledgments}

This research is an ICASE studentship award, supported by EPSRC and LAING O'ROURKE PLC under Grant No. 13440016. Any opinions, findings, and conclusions or recommendations included in this paper are those of the authors and do not necessarily reflect the views of organizations and people mentioned above.

\section{References}

[1] E.C. Lim, The analysis of productivity in building construction, Doctoral Thesis, Loughborough University, 1996. https://dspace.lboro.ac.uk/2134/7150.

[2] M.J. Horman, R. Kenley, Quantifying Levels of Wasted Time in Construction with, J. Constr. Eng. Manag. 131 (2005) 52-61.

[3] H.E. Picard, Driving down construction project labor cost, J. Constr. Manag. Assoc. Am. (2004) $1-10$.

[4] S.P. Dozzi, S.M. AbourRizk, Productivity in Construction, Ottawa: Institute for Research in Construction, National Research Council, 1993.

[5] M.E. Shehata, K.M. El-Gohary, Towards improving construction labor productivity and projects' performance, Alexandria Eng. J. 50 (2011) 321-330.

[6] V. Carrasco, B. Hall, J. Sweany, Labor and Productivity Analysis, Denver International Airport - South Terminal Redevelopment Program, 2013.

[7] AMAC Consultants, Productivity Measurement and Analysis, The University of British Columbia, 2004.

[8] J. Hildreth, M. Vorster, J. Martinez, Reduction of Short-Interval GPS Data for Construction Operations Analysis, J. Constr. Eng. Manag. 131 (2005) 920-927.

[9] H. Jiang, P. Lin, M. Qiang, Q. Fan, A labor consumption measurement system based on real-time tracking technology for dam construction site, Autom. Constr. 52 (2015) 115.

[10] T. Cheng, M. Venugopal, J. Teizer, P. a. Vela, Performance evaluation of ultra wideband technology for construction resource location tracking in harsh environments, Autom. Constr. 20 (2011) 1173-1184.

[11] A.J. Sedehi, Leveraging radio frequency technology identification for productivity analysis in high-rise construction, M.S. Thesis, Georgia Institute of Technology, 2010.

[12] R. Navon, E. Goldschmidt, Can Labor Inputs be Measured and Controlled Automatically?, J. Constr. Eng. Manag. 127 (2003) 437-445.

[13] T. Cheng, J. Teizer, G.C. Migliaccio, U.C. Gatti, Automated task-level activity analysis through fusion of real time location sensors and worker's thoracic posture data, Autom. Constr. 29 (2013) 24-39.

[14] E. Nasr, T. Shehab, A. Vlad, Tracking Systems in Construction : Applications and Comparisons, in: 49th ASC Annu. Int. Conf. Proc., 9-13 April 2013, San Luis Obispo, California, USA, 2013.

[15] A. Juels, RFID security and privacy: a research survey, IEEE J. Sel. Areas Commun. 24 (2006) 381-394.

[16] M. Bügler, G. Ogunmakin, J. Teizer, P.A. Vela, A. Borrmann, A Comprehensive Methodology for Vision-Based Progress and Activity Estimation of Excavation Processes for Productivity Assessment, in: EG-ICE, Cardiff, United Kingdom, 2014: pp. 1-10.

[17] J. Gong, C.H. Caldas, Computer Vision-Based Video Interpretation Model for Automated Productivity Analysis of Construction Operations, J. Comput. Civ. Eng. ASCE. 24 (2010) 252-263.

[18] J. Gong, C.H. Caldas, An object recognition, tracking, and contextual reasoning-based video interpretation method for rapid productivity analysis of construction operations, Autom. Constr. 20 (2011) 1211-1226.

[19] J. Yang, P. Vela, J. Teizer, Z. Shi, Vision-Based Tower Crane Tracking for Understanding Construction Activity, J. Comput. Civ. Eng. 28 (2014) 103-112.

[20] D.W. Halpin, L.S. Riggs, Planning and Analysis of Construction Operations, John Wiley \& Sons, 1992.

[21] J.C. Martinez, P.G. Ioannou, General purpose simulation with stroboscope, in: Winter Simul. Conf., Orlando, Florida, 1994.

[22] M. Golparvar-Fard, A. Heydarian, J.C. Niebles, Vision-based action recognition of earthmoving 
equipment using spatio-temporal features and support vector machine classifiers, Adv. Eng. Informatics. 27 (2013) 652-663.

[23] J. Zou, H. Kim, Using Hue , Saturation , and Value Color Space for Hydraulic Excavator Idle Time Analysis, J. Comput. Civ. Eng. 21 (2007) 238-246.

[24] J. Yang, Z. Shi, Z. Wu, Vision-based action recognition of construction workers using dense trajectories, Adv. Eng. Informatics. 30 (2016) 327-336.

[25] Y. Bai, J. Huan, A. Peddi, Development of Human Poses for the Determination of On-site Construction Productivity in Real-time, Final Report, National Science Foundation, 90 pgs, 2008

[26] A. Khosrowpour, I. Fedorov, A. Holynski, C. Niebles, M. Golparvar-Fard, Automated Worker Activity Analysis in Indoor Environments for Direct-Work Rate Improvement from long sequences of RGB-D Images, in: Constr. Res. Congr. 2014, 2014: pp. 140-149.

[27] N. Dalal, B. Triggs, Histograms of Oriented Gradients for Human Detection, in: IEEE Comput. Soc. Conf. Comput. Vis. Pattern Recognit., 2005: pp. 886-893.

[28] W. Abu-Ain, S.N.H.S. Abdullah, B. Bataineh, T. Abu-Ain, K. Omar, Skeletonization Algorithm for Binary Images, Procedia Technol. 11 (2013) 704-709.

[29] R.G. Brereton, G.R. Lloyd, Support Vector Machines for classification and regression, Analyst,. 135 (2010) 230-267.

[30] I.N. da Silva, D. Hernane Spatti, R. Andrade Flauzino, L.H.B. Liboni, S.F. dos Reis Alves, Artificial Neural Network Architectures and Training Processes, in: Artif. Neural Networks, Springer International Publishing, Switzerland, 2017: pp. 21-29.

[31] U.C. Gatti, G.C. Migliaccio, S.M. Bogus, S. Schneider, An exploratory study of the relationship between construction workforce physical strain and task level productivity, Constr. Manag. Econ. 32 (2014) 548-564.

[32] R. Akhavian, A.H. Behzadan, Smartphonebased construction workers' activity recognition and classification, Autom. Constr. 71 (2016) 198-209.

[33] J. Chen, J. Qiu, C. Ahn, Construction worker's awkward posture recognition through supervised motion tensor decomposition, Autom. Constr. 77 (2017) 67-81.

[34] C.-F. Cheng, A. Rashidi, M.A. Davenport, D. V. Anderson, Automation in Construction Activity analysis of construction equipment using audio signals and support vector machines, Autom. Constr. 81 (2017) 240-253.

[35] I.P.T. Weerasinghe, J.Y. Ruwanpura, Automated Multiple Objects Tracking System (AMOTS), in: Constr. Res. Congr., May 8-10, Banff, Alberta, Canada, 2010: pp. 11-20.

[36] E. Konstantinou, I. Brilakis, 3D Matching of Resource Vision Tracking Trajectories, in: Constr. Res. Congr., June 2016, San Juan, Puerto Rico, 2016.

[37] R.I. Hartley, In defence of the 8-point algorithm, Proc. IEEE Int. Conf. Comput. Vis. 19 (1997) 1064-1070. doi:10.1109/ICCV.1995.466816.

[38] M.M. Cundecha, Study of Factors Affecting Labor Productivity at a Building Construction Project in the USA : Web Survey, M.S. Thesis, North Dakota University, 2012.

[39] K.M. El-gohary, R.F. Aziz, Factors Influencing Construction Labor Productivity in Egypt, J. Manag. Eng. (2014) 1-9.

[40] A.M. Jarkas, C.G. Bitar, Factors Affecting Construction Labor Productivity in Kuwait, J. Constr. Eng. Manag. (2012) 811-820.

[41] C.J.O. Kuykendall, Key factors affeting labor productivity in the construction, M.S. Thesis, University of Florida, 2007.

[42] E. Lim, J. Alum, Construction productivity: Issues encountered by contractors in Singapore, Int. J. Proj. Manag. 13 (1995) 51-58.

[43] A. Makulsawatudom, M. Emsley, K. Sinthawanarong, Critical Factors Influencing Construction Productivity in Thailand, J. KMITNB. 14 (2004) 1-6. 